\title{
TITLE:
}

\section{A class of steady solutions to two- dimensional free convection}

AUTHOR(S):

Toh, S; Matsumoto, T

CITATION:

Toh, S ... [et al]. A class of steady solutions to two-dimensional free convection. PHYSICS OF FLUIDS 2003, 15(11): 3385-3396

ISSUE DATE:

2003-11

URL:

http://hdl.handle.net/2433/50015

\section{RIGHT:}

Copyright 2003 American Institute of Physics. This article may be downloaded for personal use only. Any other use requires prior permission of the author and the American Institute of Physics. 


\title{
A class of steady solutions to two-dimensional free convection
}

\author{
Sadayoshi Toh and Takeshi Matsumoto \\ Division of Physics and Astronomy, Graduate School of Science, Kyoto University, Kyoto 606-8502, Japan
}

(Received 22 April 2002; accepted 23 July 2003; published 22 September 2003)

\begin{abstract}
We obtained steady solutions to the two-dimensional Boussinesq approximation equations without a mean temperature gradient. This system is referred to as free convection in this paper. Under an external flow described by the stream function $\Psi=-A y f(x)$, steady solutions are found. They are kept steady by the balance between the strain of $\Psi$ and the diffusion. In this sense, they are similar to the Burgers vortex layer solution. Two examples other than $f(x)=x$ are shown to have steady solutions. We discuss the relation between these solutions and long-lived fine scale coherent structures observed in direct numerical simulations of two-dimensional free convection turbulence. (c) 2003 American Institute of Physics. [DOI: 10.1063/1.1613643]
\end{abstract}

\section{INTRODUCTION}

In the incompressible three-dimensional Navier-Stokes (3-DNS) turbulence, it is observed that high vorticity regions take the form of long-lived slender tube-like structures, which are called coherent structures. ${ }^{1}$ These vortex filaments are well-known to be approximated locally by the Burgers vortex tube, a steady solution to 3-DNS equations. In passive scalar turbulence, scalar also forms coherent structures. ${ }^{2,3}$ One of the important issues of the study of turbulence is to understand the dynamics of coherent structures, their role on the overall turbulence dynamics and their significance on the statistical properties of the turbulence. ${ }^{1}$ For the moment there are still long ways to go for these goals.

In this paper we consider an active scalar system that is a two-dimensional Boussinesq convection model without a mean temperature gradient in a doubly periodic square domain, which is called two-dimensional free convection (2DFC). There are two remarkable similar points between 2-DFC and 3-DNS turbulence: cascade and the appearance of coherent structures. About the cascade of 2-DFC, it has been shown that the temperature variance, called entropy, cascades from larger scales to smaller scales in 2-DFC when the turbulent state is maintained by a temperature forcing., The filament-like coherent structures seen in a direct numerical simulation (DNS) of 2-DFC turbulence are shown in Fig. 1. They are long-lived sharp interfaces between hot and cold regions, i.e., shock fronts. The typical length and width of the structures are the order of the integral scale and the order of Kolmogorov dissipation length scale. Furthermore, the evolution equations for the key quantities of the coherent structures, i.e., vorticity for 3-DNS and gradient of temperature for 2-DFC, have the same stretching terms.

Based on this similarity, in spite of the difference of space dimension between 2-DFC and 3-DNS, we expect parallelism between 2-DFC and 3-DNS. The purposes of this paper are (i) to model the coherent structures of 2-DFC with a class of steady solutions of 2-DFC equations as done in the 3 -DNS with the Burgers vortex solutions, and (ii) to check validity of the model by comparison with the DNS data of 2-DFC. We present evidence that coherent structures of
2-DFC are well approximated by the steady solutions to be considered here.

However, there is a crucial difference about coherent structures between 2-DFC and 3-DNS: the coherent structures in 2-DFC are identical to those of the local temperature dissipation rate. The coherent structures in 2-DFC can relate to the entropy cascade in a direct manner. Furthermore, recent studies about two-dimensional convection in a periodic domain by several groups revealed an interesting relation between the statistical behavior of temperature and the coherent structures: the exponents of temperature structure functions calculated by DNS saturate as the order goes to infinity as observed in passive scalar turbulence. ${ }^{4}$ This behavior is regarded as a signature of coherent structures like sharp shocks between hot and cold regions. ${ }^{7,8}$

The equations of 2-DFC are

$$
\begin{aligned}
& \partial_{t} T+(\mathbf{u} \cdot \boldsymbol{\nabla}) T=\kappa \Delta T, \\
& \partial_{t} \mathbf{u}+(\mathbf{u} \cdot \boldsymbol{\nabla}) \mathbf{u}=-\frac{\boldsymbol{\nabla} p}{\rho_{0}}+\alpha g T \hat{\mathbf{e}}+\nu \Delta \mathbf{u}, \\
& \boldsymbol{\nabla} \cdot \mathbf{u}=0 .
\end{aligned}
$$

Here $\kappa, \rho_{0}, \alpha, g$ and $\nu$ are the molecular diffusivity, the mean density of the fluid (we take $\rho_{0}$ to be unity for simplicity), the thermal expansion coefficient, the gravitational acceleration and the kinematic viscosity, respectively. The vector $\mathbf{e}$ is the unit vector in the direction opposite to the gravity. In the DNS of 2-DFC, to keep the system statistically stationary, a large-scale forcing term is added to Eq. (1) and a large-scale friction term is added to Eq. (2). To deal with temperature shocks more directly, we use the following vector quantity: ${ }^{9,10}$

$$
\chi \equiv\left(\partial_{y} T,-\partial_{x} T\right),
$$

which is called $T$-vorticity here. $T$-vorticity obeys the equation

$$
\partial_{t} \boldsymbol{\chi}+(\mathbf{u} \cdot \boldsymbol{\nabla}) \boldsymbol{\chi}=(\boldsymbol{\chi} \cdot \boldsymbol{\nabla}) \mathbf{u}+\kappa \Delta \boldsymbol{\chi},
$$

which is quite similar to the three-dimensional vorticity equation. Thus $T$-vorticity plays an essential role in 2-DFC 


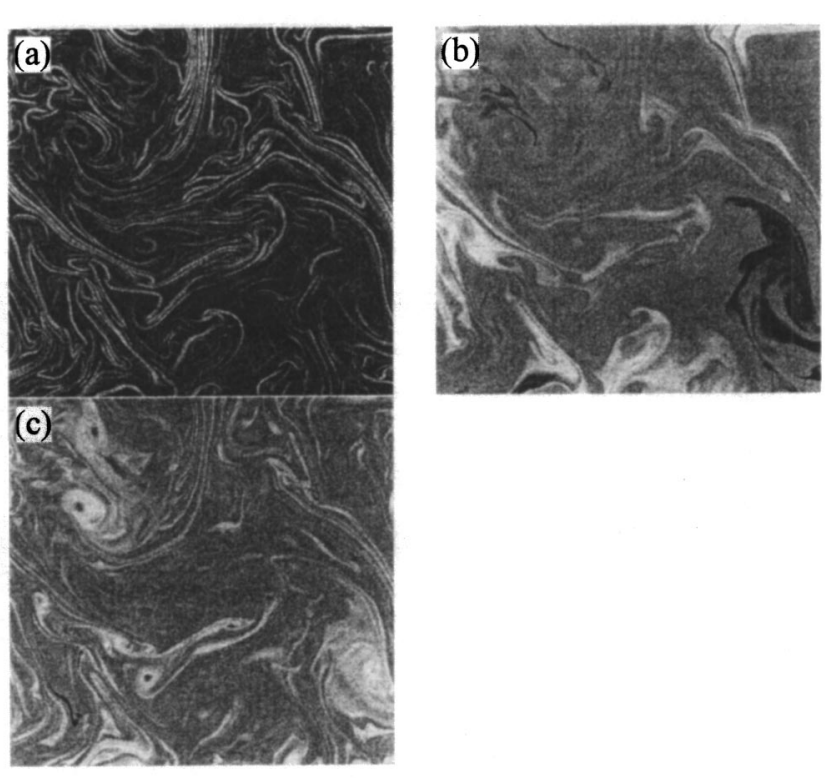

FIG. 1. (a) Modulus of the temperature gradient of a snapshot obtained by a pseudo-spectral direct numerical simulation of 2-DFC with $2048^{2}$ grid points in a periodic domain $[0,2 \pi]^{2}$. The brighter region has larger value. High temperature gradient regions, which are equivalent to high local temperature dissipation rate regions, form a long and slender structure. Many coherent structures form bundles together. The figure shows one quarter of the whole computational domain. The direction of the gravitational force is from top to bottom. The details of the DNS are in Sec. II B. (b) The temperature of the same snapshot. Plumes of various scales are seen. (c) The vorticity of the same snapshot. It is noted that vorticity also forms slender structures that correspond to shears caused by strong temperature differences at the coherent structures shown in Fig. 1(a). This behavior is completely different from that of the barotropic two-dimensional turbulence.

like vorticity in 3-DNS. In contrast with $T$-vorticity, the governing equation for vorticity $\omega(x, y, t)$ in 2 -DFC can be written as

$$
\partial_{t} \omega+(\mathbf{u} \cdot \boldsymbol{\nabla}) \omega=\alpha g\{\boldsymbol{\nabla} \times(T \hat{\mathbf{e}})\}_{z}+\nu \Delta \omega .
$$

Here $\{\cdot\}_{z}$ denotes taking the $z$-component. Note that because of the buoyancy term, i.e., the first term on the r.h.s. of Eq. (6), vorticity is not a conservative quantity even for $\nu=0$. For simplicity, we assume $\nu=\kappa$, i.e., Prandtl number $\operatorname{Pr}$ $\equiv \nu / \kappa=1$ here.

In the following sections, we consider steady solutions to Eqs. (5) and (6), where the flow is decomposed into the external part given by a stream function $\Psi=-A y f(x)$ and a response to it. In Sec. II, we deal with the case $f(x)=x$, i.e., a stagnation flow, and compare the obtained steady solutions with coherent structures seen in the DNS of 2-DFC. In Sec. III, extended stagnation flows $f(x) \neq x$ are considered. We show two examples of $f(x)$. The motivation of this extension is to model bundles of the coherent structures as seen in Fig. 1(a). The realizability of the extended external flow is also discussed. Concluding remarks are made in Sec. IV.

\section{BURGERS VORTEX LAYER SOLUTIONS}

We assume that the system, Eqs. (5) and (6), is exposed to a stagnation flow,

$$
\Psi(x, y)=-A x y,
$$

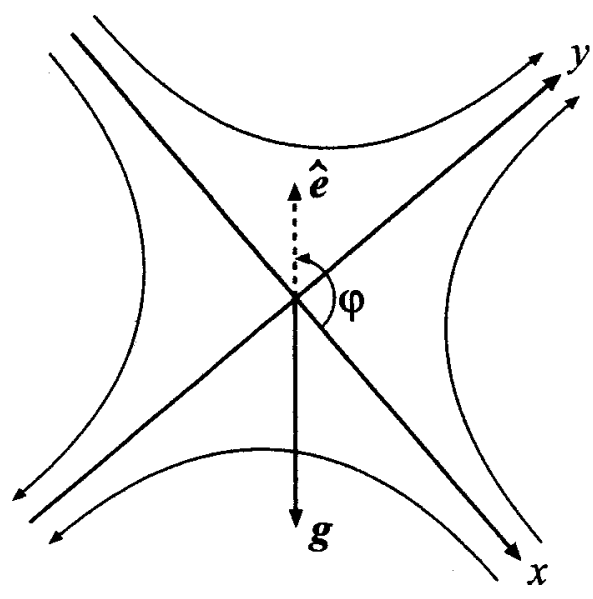

FIG. 2. The coordinate system fixed on the external stagnation flow. The direction of the gravity vector $\mathbf{g}$ is expressed via the angle $\varphi$.

where $A$ is a positive constant so that we take the directions of contraction and expansion as the $x$-axis and $y$-axis, respectively. The angle between the $x$-axis and $\hat{\mathbf{e}}$ is also a parameter and denoted by $\varphi$ as shown in Fig. 2. Then the unit vector $\hat{\mathbf{e}}$ in Eq. (1) is given in this coordinate as $\hat{\mathbf{e}}$ $=(\cos \varphi, \sin \varphi)$. We use this coordinate throughout this paper. We further assume the following forms of the temperature and velocity fields:

$$
\begin{aligned}
T(x, y, t) & =\theta(x), \\
\mathbf{u}(x, y, t) & =\left(\partial_{y} \Psi(x, y),-\partial_{x} \Psi(x, y)\right)+(0, v(x)) \\
& =(-A x, A y+v(x)) .
\end{aligned}
$$

In terms of $\theta$ and $v, T$-vorticity and vorticity are expressed as

$$
\begin{aligned}
& \chi(x, y, t)=\left(0,-\partial_{x} \theta(x)\right) \equiv\left(0, \chi_{y}(x)\right), \\
& \omega(x, y, t)=\omega(x)=\frac{d v(x)}{d x} .
\end{aligned}
$$

Consequently, Eqs. (5) and (6) are reduced to the following ordinary differential equations:

$$
\begin{aligned}
& \frac{d}{d x}\left(\frac{\kappa}{A} \frac{d \chi_{y}}{d x}+x \chi_{y}\right)=0, \\
& \frac{\nu}{A} \frac{d^{2} \omega}{d x^{2}}+x \frac{d \omega}{d x}=\chi_{y} \frac{\alpha g \sin \varphi}{A} .
\end{aligned}
$$

After integrating once, Eq. (12) can be re-written as

$$
\frac{\kappa}{A} \frac{d \chi_{y}}{d x}+x \chi_{y}=0
$$

which is actually identical to Eq. (1) along with the conditions, Eqs. (8) and (9). The right hand side therefore should be zero.

Let us consider the general boundary conditions at the origin:

$$
\begin{aligned}
& \chi_{y}(x=0)=\chi_{0}, \\
& \chi_{y}^{\prime}(x=0)=0,
\end{aligned}
$$



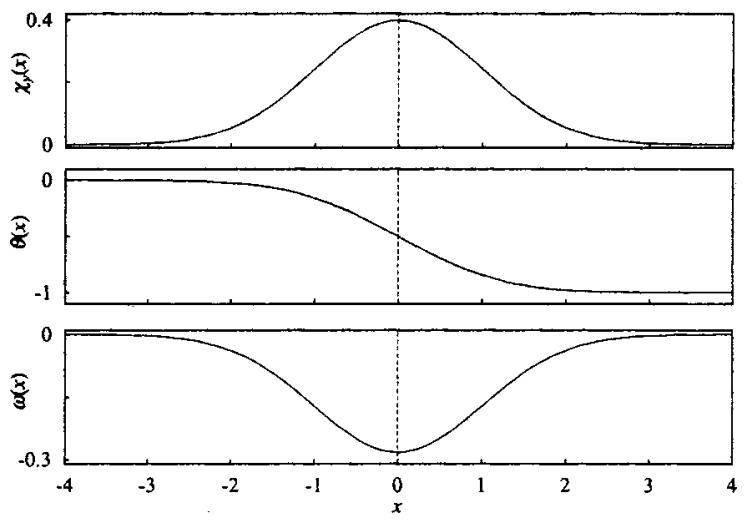

FIG. 3. The $T$-vorticity, Eq. (23), the corresponding temperature $\theta(x)$ and the vorticity, Eq. (24). Here we set the parameters as $\Theta_{0}=1, A=1, \kappa=1$, $\alpha g=1$ and $\varphi=\pi / 4$.

$$
\begin{aligned}
& \omega(x=0)=\omega_{0}, \\
& \omega^{\prime}(x=0)=\omega_{0}^{\prime},
\end{aligned}
$$

where $\omega^{\prime}(x)$ denotes the derivative of vorticity; $\chi_{0}, \omega_{0}$ and $\omega_{0}^{\prime}$ are constants. With $\kappa=\nu$ the solutions to Eqs. (12) and (13) are obtained as

$$
\begin{aligned}
\chi_{y}(x)= & \chi_{0} e^{-(A / 2 \kappa) x^{2}}, \\
\omega(x)= & \omega_{0}+\omega_{0}^{\prime} \int_{0}^{x} e^{-(A / \kappa) \xi^{2}} d \xi+\chi_{0} \frac{\alpha g \sin \varphi}{A} \\
& \times\left(1-e^{-(A / 2 \kappa) x^{2}}\right) .
\end{aligned}
$$

Here only the last term of Eq. (20) is the buoyancy generated vorticity which is set to null at $x=0$. The first two are homogeneous solutions, i.e., the solutions to the vorticity equation Eq. (13) setting the r.h.s. (the buoyancy force) zero.

\section{A. Burgers vortex layer solutions}

The boundary conditions to be considered here are

$$
\begin{aligned}
& \chi_{y}(x) \rightarrow 0 \quad(x \rightarrow \pm \infty), \\
& \omega(x) \rightarrow 0 \quad(x \rightarrow \pm \infty),
\end{aligned}
$$

which are equivalent to setting $\omega_{0}=-\chi_{0} \alpha g \sin \varphi / A$ and $\omega_{0}^{\prime}$ $=0$. The general solutions, Eqs. (19) and (20), are then reduced to the Burgers vortex layer solutions,

$$
\begin{aligned}
\chi_{y}(x) & =\Theta_{0} \sqrt{\frac{A}{2 \pi \kappa}} \exp \left(-\frac{A}{2 \kappa} x^{2}\right), \\
\omega(x) & =-\frac{\alpha g \sin \varphi}{A} \Theta_{0} \sqrt{\frac{A}{2 \pi \kappa}} \exp \left(-\frac{A}{2 \kappa} x^{2}\right) \\
& =-\frac{\alpha g \sin \varphi}{A} \chi_{y}(x),
\end{aligned}
$$

where $\Theta_{0}=\int_{-\infty}^{\infty} \chi_{y}(\xi, t=0) \quad d \xi=\theta(-\infty, 0)-\theta(\infty, 0) . \quad$ In these solutions, the temperature difference corresponds to the circulation of the vortex layer for the barotropic case. In Fig. 3 we plot the solutions; $T$-vorticity, Eq. (23), and the corresponding temperature $\theta(x)$ and vorticity, Eq. (24). Since the temperature $\theta(x)$ is a monotonic function, $\chi_{y}(x)$ contains a single bump. We call the solutions Burgers $T$-vortex layer solutions.

Now we make several remarks on the solutions, Eqs. (23) and (24). First, we note that $\omega(x)$ is proportional to $\chi_{y}(x)$ and $\sin \varphi$. That is, $\chi_{y}(x)$ is not dependent on the direction of the gravity, but $\omega(x)$ is. This is because the $T$-vorticity solution is maintained by the balance between the imposed strain and the diffusion. On the other hand, the vorticity is maintained by the balance between the diffusion and the buoyancy induced by the temperature difference across the Burgers $T$-vortex layer. Hence the vorticity is maximized when the direction of the $T$-vortex layer coincides with that of the gravity.

Second, a characteristic time of the relaxation to the steady state, Eq. (23), for given temperature difference $\Theta_{0}$ can be estimated. Under the stagnation flow, Eq. (9), the time-dependent solution to Eq. (5) is obtained ${ }^{11}$ as

$$
\begin{aligned}
\chi_{y}(x, t)= & \left(4 \pi \kappa \frac{1-e^{-2 A t}}{2 A}\right)^{-1 / 2} \int_{-\infty}^{\infty} \chi_{y}(\xi, t=0) \\
& \times \exp \left[-\frac{\left(x-e^{-A t} \xi\right)^{2}}{4 \kappa\left(1-e^{-2 A t}\right) / 2 A}\right] d \xi \\
& \rightarrow \Theta_{0} \sqrt{\frac{A}{2 \pi \kappa}} \exp \left(-\frac{A}{2 \kappa} x^{2}\right), \text { as } t \rightarrow \infty
\end{aligned}
$$

The characteristic time for the relaxation is thus given as $1 / A$.

Third, the characteristic width of the bump of $T$-vorticity and vorticity is $\sqrt{2 \kappa / A}$. This agrees with an observation about structures of a passive scalar advected by a twodimensional synthetic random velocity field with a finite correlation time in Ref. 12. (Of course, vorticity is not considered in that case.) The problem considered in Ref. 12 is completely different from our case, i.e., a passive scalar with a nonzero mean gradient is treated besides the synthetic velocity field. However, at least locally, the equations for passive scalar and corresponding $\boldsymbol{\chi}$ are likely to hold. Hence, once a stagnation flow is realized, the passive scalar can relax to the steady solution Eq. (23). The following features reported in Ref. 12 are consistent with this view: (i) the width of the coherent structures of the passive scalar was found to scale well with $\sqrt{\kappa / s}$, where $s$ was a root mean square of the rate of strain calculated from the low-path filtered velocity and (ii) they also showed a stagnation flow associated with a coherent structure in Fig. 7 of Ref. 12.

\section{B. Comparison with coherent structures in 2-DFC turbulence}

In this subsection, we compare the Burgers vortex layer solutions, Eqs. (23) and (24), with the coherent structures observed in the DNS of 2-DFC turbulence.

Let us first mention the details of DNS. We employ a pseudo-spectral method with dealiasing through the grid shift method. The fourth order Runge-Kutta method is used for a temporal marching scheme. For realizing a statistically stationary state, we actually simulate the following equations: 
TABLE I. Numerical and flow parameters of DNS. The coefficient of the buoyancy term $\alpha g$ is set to unity for all resolutions. The kinematic viscosity $\nu$ is taken to be equal to corresponding $\kappa$. Temperature dissipation length scale $\eta_{\theta} \equiv\left[\kappa^{5} / \epsilon_{\theta}(\alpha g)^{2}\right]^{1 / 8}$, where $\epsilon_{\theta}$ is temperature dissipation rate, is the smallest length scale in the flow. The Bolgiano-Obukhov length scale is defined as $l_{\mathrm{BO}}=\epsilon^{5 / 4} /\left[\epsilon_{\theta}^{3 / 4}(\alpha g)^{3 / 2}\right]$, where $\epsilon$ is the energy dissipation rate. The two scales $l_{\mathrm{BO}}$ and $\eta_{\theta}$ are the same order of magnitude, implying that the flow is dominated by the entropy cascade.

\begin{tabular}{cccccc}
\hline \hline Grid points & $\kappa$ & $F_{0}$ & $d_{0}$ & $\eta_{\theta}$ & $l_{\text {BO }}$ \\
\hline $512^{2}$ & $5 \times 10^{-4}$ & 0.4 & 0.1 & $1.15(2 \pi / 512)$ & $3.1 \eta_{\theta}$ \\
$1024^{2}$ & $1 \times 10^{-4}$ & 0.4 & 0.1 & $0.950(2 \pi / 1024)$ & $4.8 \eta_{\theta}$ \\
$2048^{2}$ & $5 \times 10^{-5}$ & 0.4 & 0.1 & $1.26(2 \pi / 2048)$ & $4.5 \eta_{\theta}$ \\
\hline \hline
\end{tabular}

$$
\begin{aligned}
& \partial_{t} T+(\mathbf{u} \cdot \boldsymbol{\nabla}) T=\kappa \Delta T+F, \\
& \partial_{t} \omega+(\mathbf{u} \cdot \nabla) \omega=\alpha g \partial_{x} T+\nu \Delta \omega+d,
\end{aligned}
$$

where the gravitational force acts in the $-y$ direction. The two terms, the large-scale forcing term $F$ and the large-scale friction term $d$ that absorbs energy transferred from smaller scales are added to maintain the turbulence statistically stationary. The large-scale forcing term used here is timeindependent:

$$
F(\mathbf{x})=F_{0} \cos (2 x) \sin (2 y),
$$

where $F_{0}$ is a constant. The large scale friction term is written in the Fourier space as

$$
\hat{d}(\mathbf{k})=\left\{\begin{array}{l}
-\frac{d_{0}}{|\mathbf{k}|} \hat{\omega}(\mathbf{k}) \quad(0<|\mathbf{k}| \leqslant 3), \\
0 \quad \text { (otherwise) }
\end{array}\right.
$$

where $d_{0}, \mathbf{k}$ and $\hat{\omega}(\mathbf{k})$ are, respectively, a constant, the wave number vector and the Fourier mode of vorticity. The simulations are started with random initial conditions and continued for more than 100 large-scale eddy turnover times. The coherent structures shown in Fig. 1(a) are spontaneously formed. The resultant numerical and flow parameters are listed in Table I. The entropy and energy spectra of the simu-

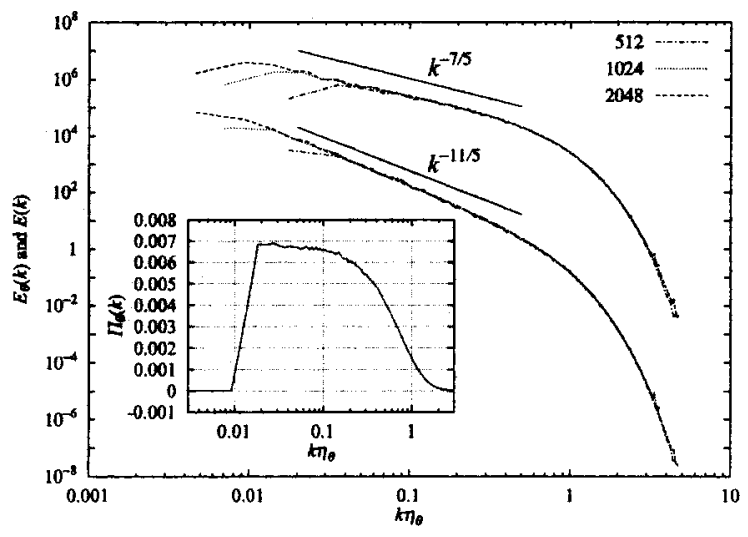

FIG. 4. Angle-averaged and normalized spectra of the temperature variance, i.e., entropy (the upper curves) and the kinetic energy (the lower curves) obtained from the DNS of three different resolutions. They are almost isotropic in the wave number space. The scalings in the inertial range of the temperature variance and the energy spectra are close to the BolgianoObukhov scalings $k^{-7 / 5}$ and $k^{-11 / 5}$, respectively. For clarity, temperature spectra are multiplied by $2.5 \times 10^{3}$. Inset: The flux function $\Pi_{\theta}(k)$ of temperature variance (entropy flux) of the DNS with $2048^{2}$ grid points. The constant entropy flux range is developed. lated flows are shown in Fig. 4. There is the inertial range in which the entropy cascades at a constant rate and the scalings of the spectra are close to the Bolgiano-Obukhov scalings. In this sense, the simulated 2-DFC turbulence exhibits similar behavior as 3-DNS turbulence.

For a comparison between the Burgers vortex layer solutions and the coherent structures of 2-DFC, a numerical identification of coherent structures is necessary. The coherent structures seen in Fig. 1(a) can be roughly regarded as thin ridges of $|\boldsymbol{\nabla} T|=|\boldsymbol{\chi}|$. Tracing a "skeleton" of a coherent structure can therefore be done by connecting a local maximum point of $|\boldsymbol{\chi}|$ to the nearest neighbor ones in the direction of the ridge. If the nearest local maximum point is farther than, say five meshes, we stop connecting the points and regard that we reach one of the end points of the structure. A second order interpolation is employed for estimating values at off-grid points in this procedure. The idea of connecting local maximum or minimum points for identification is proposed by Miura and Kida for the analysis of local pressure minimum vortex tubes in 3-DNS turbulence. ${ }^{13}$ One advantage of this method is that it does not require any threshold, i.e., some value of $|\boldsymbol{\chi}|$ in 2-DFC, for the identification. An example of the identified skeletons of 2-DFC is shown in Fig. 5.

With this identification method, we are able to extract an individual structure from a snapshot and compare it with the Burgers vortex layer solutions. In Figs. 6 and 7, we show two examples of the comparison: one is a structure with a typical length (100 to 200 meshes) in Fig. 6(a) and the other is a longer structure in Fig. 7(a).

To measure the deviation from the Burgers vortex layer solutions along the structure, the ratio,

$$
R=-\frac{A_{*} \omega\left(\mathbf{x}_{*}\right)}{\alpha g \chi_{y}^{\mathrm{DNS}}\left(\mathbf{x}_{*}\right)},
$$

is plotted in Figs. 6(b) and 7(b). Here $x_{*}$ denotes the points on the skeleton, $\chi_{y}^{\mathrm{DNS}}\left(\mathbf{x}_{*}\right)$ is the $y$-component in the DNS coordinates of $T$-vorticity at the point $\mathbf{x}_{*}$ and $A_{*}$ is the positive eigenvalue of the velocity gradient tensor at the point $\mathbf{x}_{*}$. The ratio is unity if $T$-vorticity and vorticity obeys the Burgers vortex layer solutions. [Note that $\chi_{y}^{\mathrm{DNS}}\left(\mathbf{x}_{*}\right)$ is equivalent to $\chi_{y}(x) \sin \varphi$ in Eq. (23).] In rather straight parts, the ratio is close to one, while it deviates from unity in the parts where the skeleton has large curvature. In such large curvature regions, which are dominated by rotational motion, the structures can no longer be maintained by straining mo- 

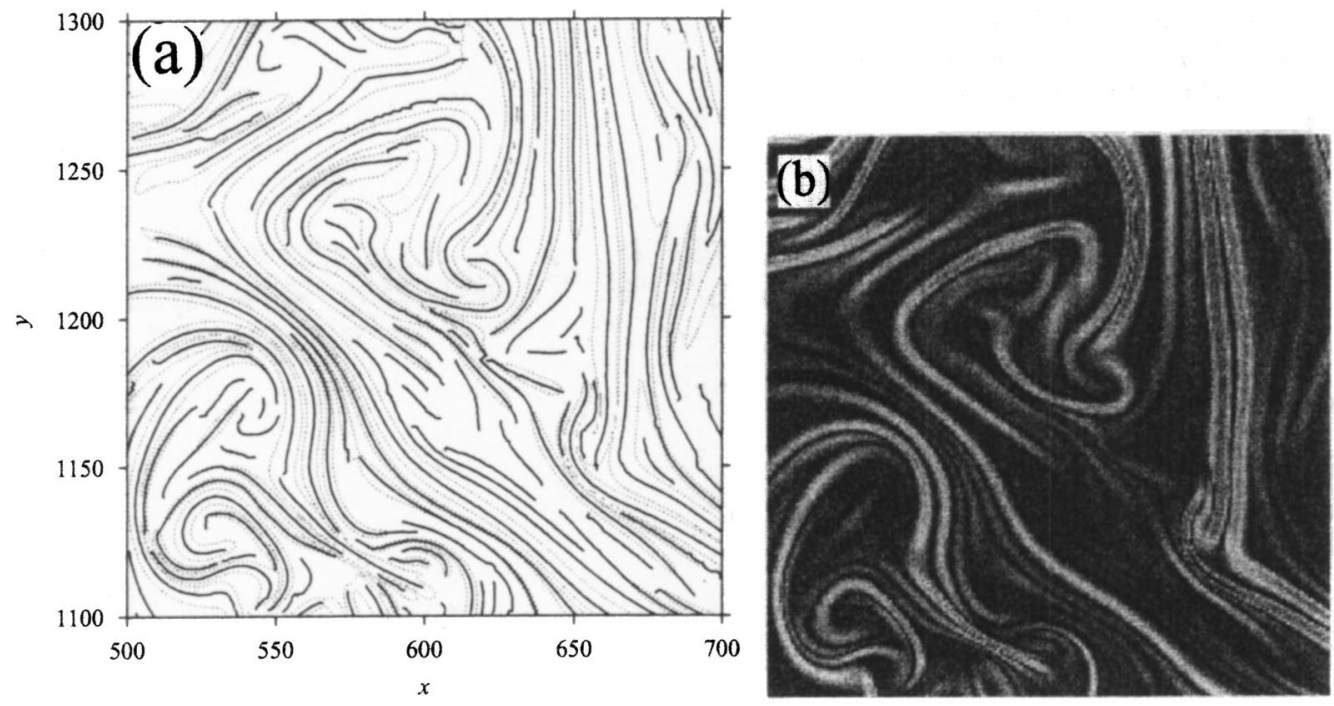

FIG. 5. (a) Numerically identified skeletons of coherent structures in the DNS. The figure contains $200^{2}$ grid points out of $2048^{2}$ points. Dotted lines are isolines of $|\boldsymbol{\chi}|$. (b) Contours of $|\boldsymbol{\chi}|$ in the corresponding region to (a). The brighter region has a larger value.

tion and split into different parts. This feature is clearly seen in the insets of Figs. 6(b) and 7(b). The places where the ratio shows divergent behavior correspond to the local minima of the modulus of $T$-vorticity along the skeleton. However, for long structures, $R=1$ regions seem not to be a large fraction of structures.

The behavior on the cut perpendicular to the skeleton is shown in Figs. 6(c) and 7(c). For a comparison with the Burgers vortex layer solutions, Eqs. (23) and (24), $T$-vorticity (two components), vorticity and the length scale are normalized by the values at the point on the skeleton. A second order interpolation is again used to estimate quantities at the off-grid points. The collapse of normalized $T$-vorticity (the component perpendicular to the cut) and vorticity to the curve $\exp \left(-s^{2}\right)$, where $s=($ length $) / \sqrt{2 \kappa / A}$, suggests that the Burgers vortex layer solutions are, at least locally, good approximations of the coherent structures. From these figures, the typical width of the structure is estimated to be a few Kolmogorov dissipation scales.

To evaluate the fraction of the sections where the Burgers vortex layer solutions give good approximations, the probability density function (pdf) of the ratio $R$ is plotted in Fig. 8. The pdf is calculated from the data on equally spaced points on each skeleton (the points where the eigenvalues of the velocity gradient tensor $A$ becomes complex are excluded). The conditional pdf on the magnitude of $T$-vorticity has a peak closer to $R=1$. This suggests that stronger $T$-vorticity structures are closer to the Burgers vortex layer solutions. For the structures having $|\boldsymbol{\chi}|>5|\boldsymbol{\chi}|_{\text {average }}$, the probability of taking the ratio $0.5 \leqslant R<1.5$ is estimated to be 0.37 . Our observation on skeletons obtained by DNS suggests that (i) the width of the structure scales well with $\sqrt{2 \kappa / A}$, where $A$ is the positive eigenvalue of the velocity gradient tensor at a point on a skeleton, (ii) the relation between $T$-vorticity and vorticity, Eq. (24), holds well in rather straight parts of structures having high $T$-vorticity.

\section{Temporal evolution of the coherent structure}

In this subsection we briefly study the temporal evolution of the coherent structure shown in Fig. 6. The purpose is to see how long the part of the structure approximated by the steady solution keeps the property. First of all, as a measure of a large scale motion, we use the definition ${ }^{14}$ of a large scale eddy turnover time $\tau$ as

$$
\tau \equiv \frac{1}{k_{*} \sqrt{\int E(k) d k}},
$$

where $k_{*}$ is the wave number at which the temperature variance (entropy) spectrum takes maximum and $E(k)$ is the energy spectrum. In our DNS with $2048^{2}$ grids points, the turnover time (31) is estimated to be $\tau \simeq 0.80\left(k_{*}=2\right)$. We track the structure for one large scale turnover time after the instance shown in Fig. 6. The evolution of the structure is shown in Figs. 9-12. From these figures it is seen that the behavior close to the stationary solutions remains for 0.2 turnover time. After this time the overall behavior of $|\boldsymbol{\chi}|$ along the structure is decay (see the insets of Figs. 10 and 12). However, it involves splitting and reconnection. The structure can be identifiable for 1.2 turnover time after the instance of Fig. 6.

To conclude this section, regarding the significance of the Burgers vortex layer solutions in the limit of vanishing diffusivity, it is well-known that the temperature dissipation rate per unit length along the $y$-axis, $\epsilon_{\theta}$, of the solution, Eq. (23), goes to zero. That is, $\epsilon_{\theta}=\int_{-\infty}^{\infty} \kappa \chi_{y}(x)^{2} d x \propto \sqrt{\kappa A} \rightarrow 0$ as $\kappa \rightarrow 0$, implying that this Burgers layer solution is irrelevant in this limit. In the DNS, however, the situation can be different because the feedback to $A$ from other ingredients of the flow should be taken into account. Indeed, the probability distribution functions of $A(x, y)$ obtained in the DNS of various values of $\kappa$ are observed to fall into a unique curve under a suitable normalization. In other words, $A(x, y)$ field may 

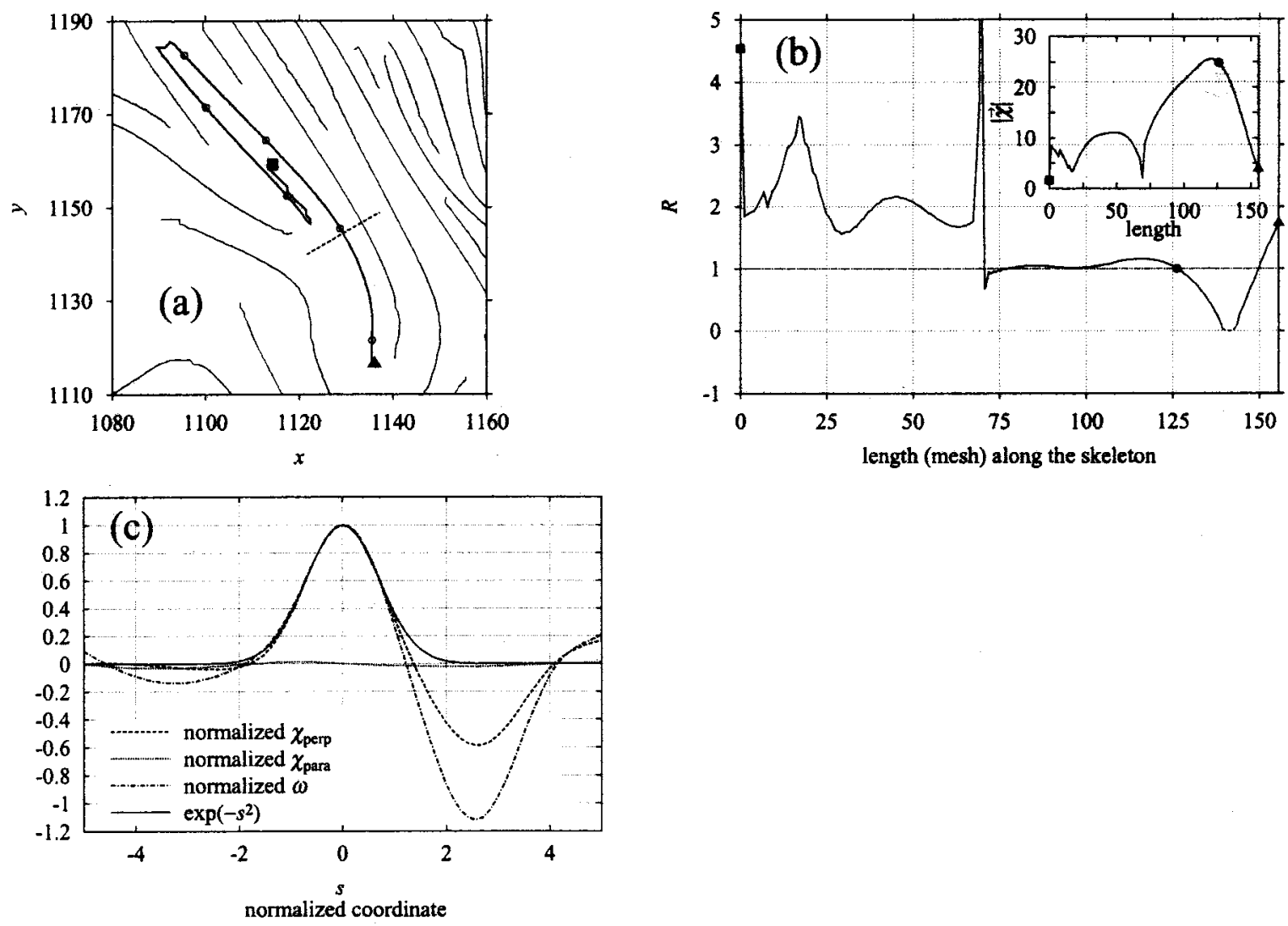

FIG. 6. (a) A tracked skeleton of a typical coherent structure obtained from the DNS with $2048^{2}$ grid points. The length of the skeleton is $0.07 \times 2 \pi$. Open circles are marked at every other 25 mesh sizes along the skeleton from the end point marked with a filled square. (b) Deviation from the Burgers vortex layer solution along the skeleton. The end points marked with a filled square and triangle correspond to the same marks in (a). The ratio $R$ is unity if $T$-vorticity and vorticity obey the Burgers vortex layer solutions. In the part denoted by the dotted line around (length) $=135$, the eigenvalue of the velocity gradient tensor becomes complex. Inset: the corresponding $|\boldsymbol{\chi}|$ along the skeleton. The spatial average of $|\boldsymbol{\chi}|$ in this snapshot is 8.1. This structure has a relatively large $|\boldsymbol{\chi}|$ value. (c) The behavior of $T$-vorticity and vorticity on the cut perpendicular to the skeleton, which is shown as the dashed line in Fig. 6(a) and the filled circle in (b). The plotted two component of $T$-vorticity, $\chi_{\text {perp }}$ and $\chi_{\text {para }}$, are perpendicular and parallel component to the cut. The origin, $s=0$, corresponds to the point on the skeleton. The coordinate $s, T$-vorticity $\chi_{\text {perp }}, \chi_{\text {para }}$ and vorticity $\omega$ are normalized with the values at the origin so that they collapse to $\exp \left(-s^{2}\right)$ if they are the Burgers vortex layer solutions, Eqs. (23) and (24). The normalized unit length scale amounts to $0.96(2 \pi / 2048)$. A width of the structure is hence estimated to be a few Kolmogorov dissipation scales (see Table I).

have some nontrivial dependency on the diffusivity $\kappa$. Thus it is conjectured that the dissipation rate of the solution Eq. (23), $\sqrt{\kappa A}$, would take a nonzero value in the limit of vanishing diffusivity in real flows. Hence, the relevance of the Burgers vortex layer solutions might not be ruled out. The detail of the observation on $A(x, y)$ field in DNS will be reported elsewhere.

The visualization of DNS flow, Fig. 1(a), shows the collective behavior of coherent structures: long structures tend to align each other. In the next section we explore the possibility of modeling these bundles of structures with steady solutions.

\section{EXTENSION}

Let us first look at a bundle of structures obtained in the DNS. A snapshot is shown in Fig. 13. The cut plotted in Fig. 13(b) is chosen to go across high $T$-vorticity structures. [As seen in Fig. 13(c) the typical magnitude of $T$-vorticity of the structures is 20, whereas the spatial average of $|\boldsymbol{\chi}|$ in this snapshot is 6.65.] One feature of this cut is that the temperature roughly decreases from one end to the other. In such a case, the staircase-like change of temperature can lead to stripes of structures. This is the point we try to model in this section. Needless to say that it is just one mechanism to cause bundles of structure. [The temperature can only change monotonically in our consideration because of the constraint that the right hand side of Eq. (14) vanishes.]

For modeling a bundle of several structures with steady solutions, we consider the external flow given by

$$
\Psi(x, y)=-A y f(x),
$$

where $f(x)$ is a function satisfying a condition mentioned later. In the previous section we treat the case $f(x)=x$. A major difference from the previous case is that the external flow, Eq. (32), has, in general, nonzero vorticity,

$$
\Omega(x, y) \equiv-\Delta \Psi=A y f^{\prime \prime}(x) .
$$

Thus $\Omega$ should satisfy the vorticity equation. The realizability of Eq. (32) will be discussed in detail later in this section.

Under the extended stagnation flow, Eq. (32), we further assume the dependence of temperature and velocity on the spatial coordinate as follows: 

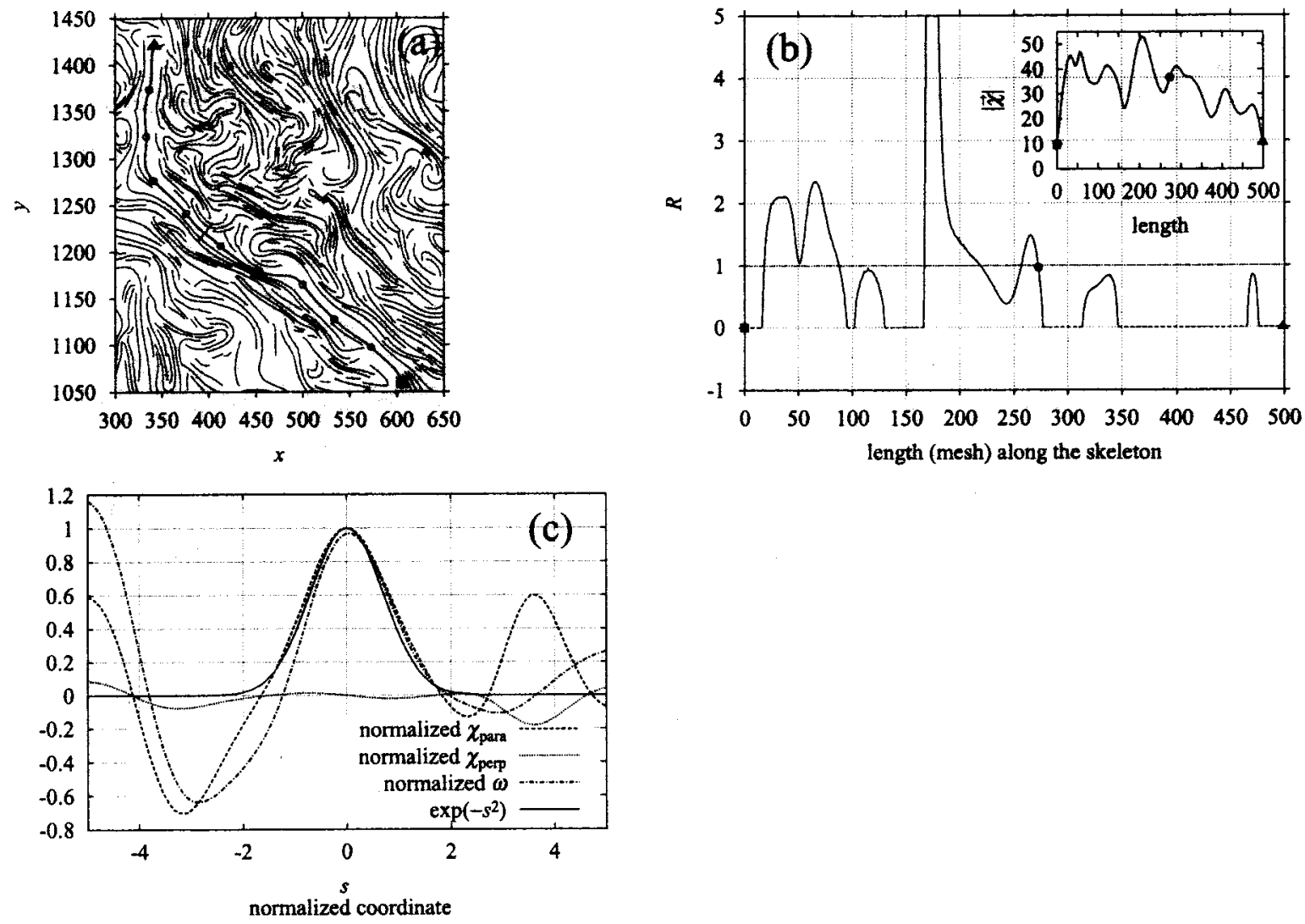

FIG. 7. (a) A tracked skeleton of a typical long structure obtained from the same snapshot as Fig. 6 . The length of the skeleton is $0.24 \times 2 \pi$. Open circles are marked at every other 50 mesh sizes along the skeleton from the end point marked with a filled square. (b) The same as Fig. 6(b) but for the longer structure. Here the fraction of the part where the eigenvalue of the velocity gradient tensor becomes complex is increased. Inset: corresponding $|\boldsymbol{\chi}|$ along the skeleton. (c) The same as Fig. 6(c) but for the longer structure. The normalized unit length scale in this figure is $1.7(2 \pi / 2048)$. A width of the structure is again estimated to be a few Kolmogorov dissipation scales (see Table I).

$$
\begin{aligned}
T(x, y, t) & =\theta(x), \\
\mathbf{u}(x, y, t) & =\left(\partial_{y} \Psi,-\partial_{x} \Psi\right)+(0, v(x)) \\
& =\left(-A f(x), A y f^{\prime}(x)+v(x)\right) .
\end{aligned}
$$

Then $\mathcal{\chi}$ and the total vorticity $\omega$ are given by

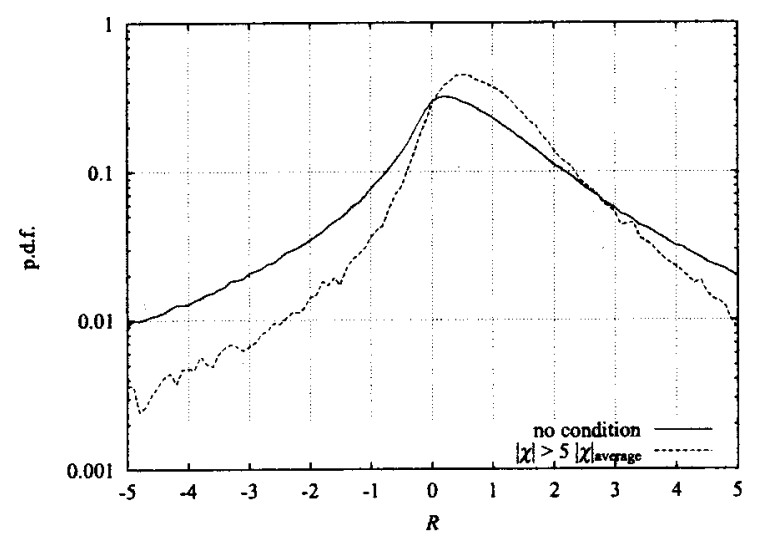

FIG. 8. Probability density functions of the ratio $R$ calculated from the DNS with $2048^{2}$ grid points. The Burgers vortex layer solutions correspond to $R=1$. The conditional probability distribution has a peak near $R=1$, i.e., the structures having larger $T$-vorticity are closer to the Burgers vortex layer solutions. The average of $T$-vorticity $|\boldsymbol{\chi}|_{\text {average }}$ is the spatial average over all grid points.

$$
\begin{aligned}
& \chi(x)=\left(0,-\theta^{\prime}(x)\right) \equiv\left(0, \chi_{y}(x)\right), \\
& \omega(x, y)=-\Delta \Psi+v^{\prime}(x) \equiv \Omega(x, y)+\widetilde{\omega}(x) .
\end{aligned}
$$

We call $\widetilde{\omega}$ response vorticity here. The velocity field, Eq. (35), is uniquely decomposed into the $y$-dependent and the $y$-independent parts.

Imposing the general boundary conditions,

$$
\begin{aligned}
& \chi_{y}(x=0)=\chi_{0}, \\
& \chi_{y}^{\prime}(x=0)=0, \\
& \widetilde{\omega}(x=0)=\widetilde{\omega}_{0}, \\
& \widetilde{\omega}^{\prime}(x=0)=\widetilde{\omega}_{0}^{\prime},
\end{aligned}
$$

steady solutions to Eqs. (5) and (6) are easily obtained for any $f(x)$ as

$$
\begin{aligned}
\chi_{y}(x) & =\chi_{0} e^{-(A / \kappa) \int_{0}^{x} f(\lambda) d \lambda}, \\
\widetilde{\omega}(x)= & \widetilde{\omega}_{0}+\widetilde{\omega}_{0}^{\prime} \int_{0}^{x} e^{-(A / \nu) \int_{0}^{\zeta} f(\lambda) d \lambda} d \zeta \\
& +\chi_{0} \frac{\alpha g \sin \varphi}{\kappa} \int_{0}^{x} \zeta e^{-(A / \nu) \int_{0}^{\zeta} f(\lambda) d \lambda} d \zeta .
\end{aligned}
$$



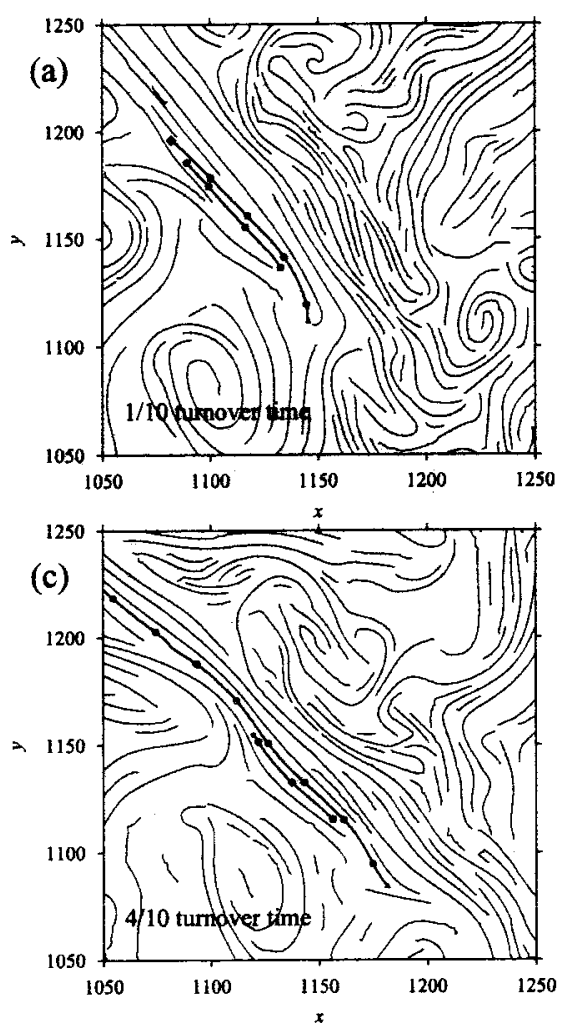

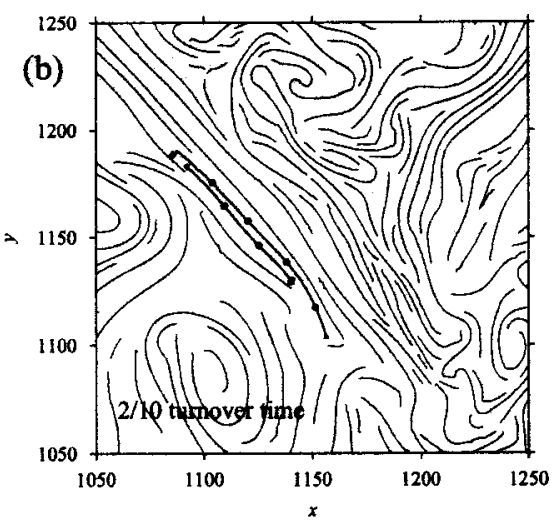

FIG. 9. The early stage evolution of the structure shown in Fig. 6. The longer structure in (b) reconnects to a top-right neighboring structure at 0.38 turnover time after the instance of Fig. 6.
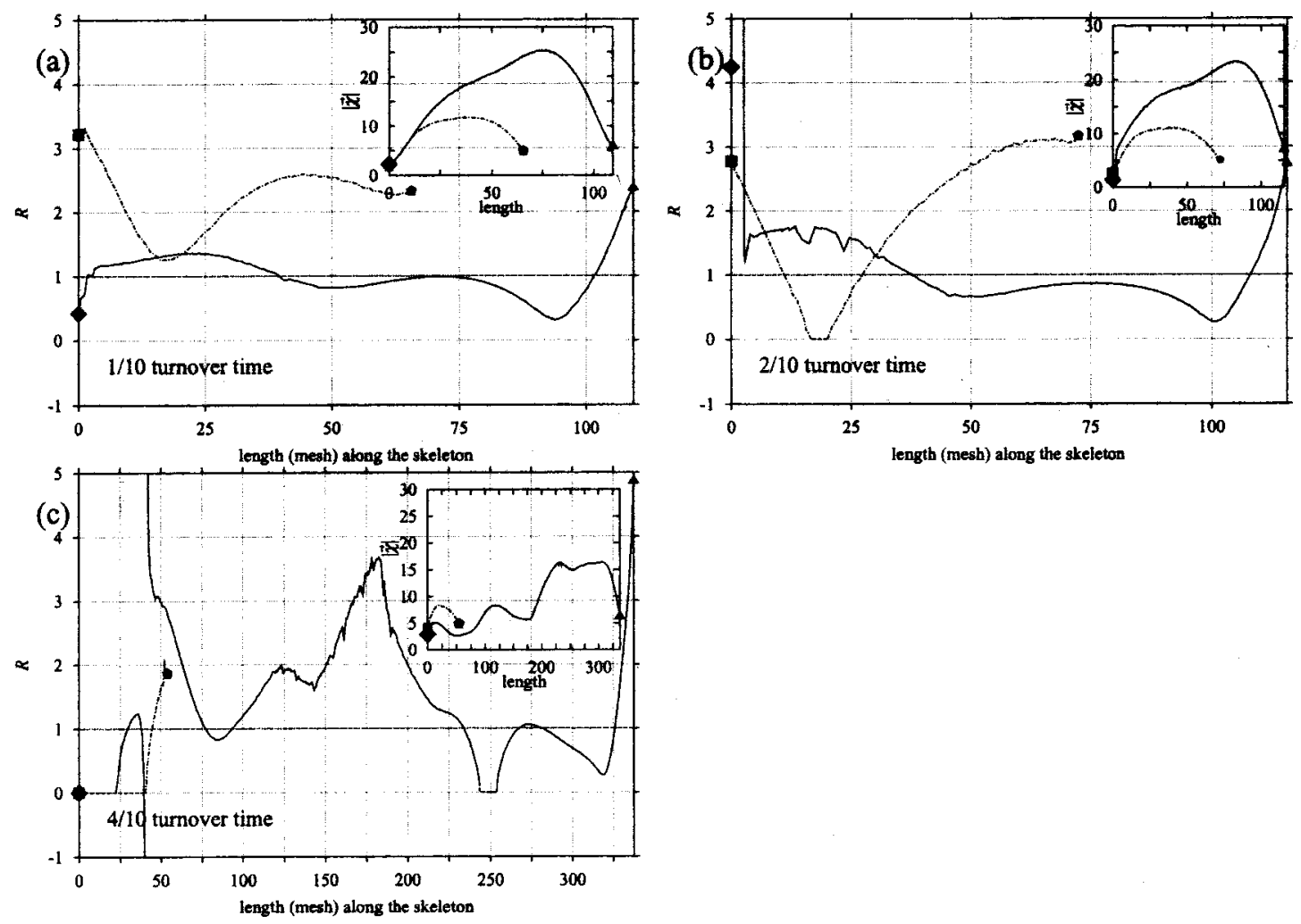

FIG. 10. The early stage evolution of the ratio $R$ of the structure shown in Fig. 6. In (c), the length of the longer structure (denoted by the solid line) changes abruptly due to the reconnection. Only the right end in (c) corresponds to the tracked structure shown (a) and (b). Insets: corresponding modulus of $T$-vorticity, $|\boldsymbol{\chi}|$. The modulus decays rapidly between 0.2 and 0.4 turnover time. 

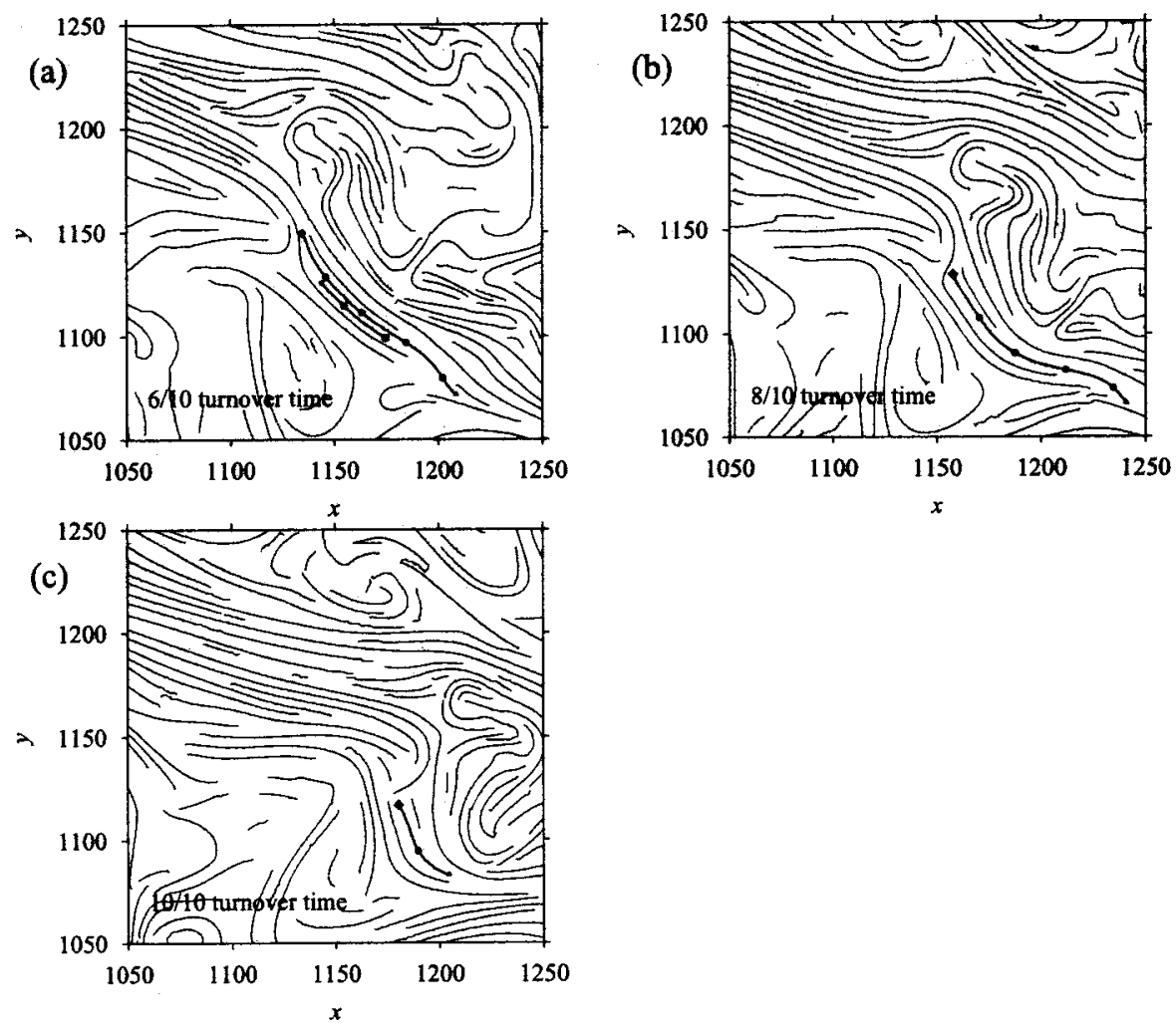

FIG. 11. The later stage evolution of the structure shown in Fig. 6. The longer structure in (a) splits already at 0.51 turnover time. The shorter structure in (a) decays out at 0.67 turnover time.
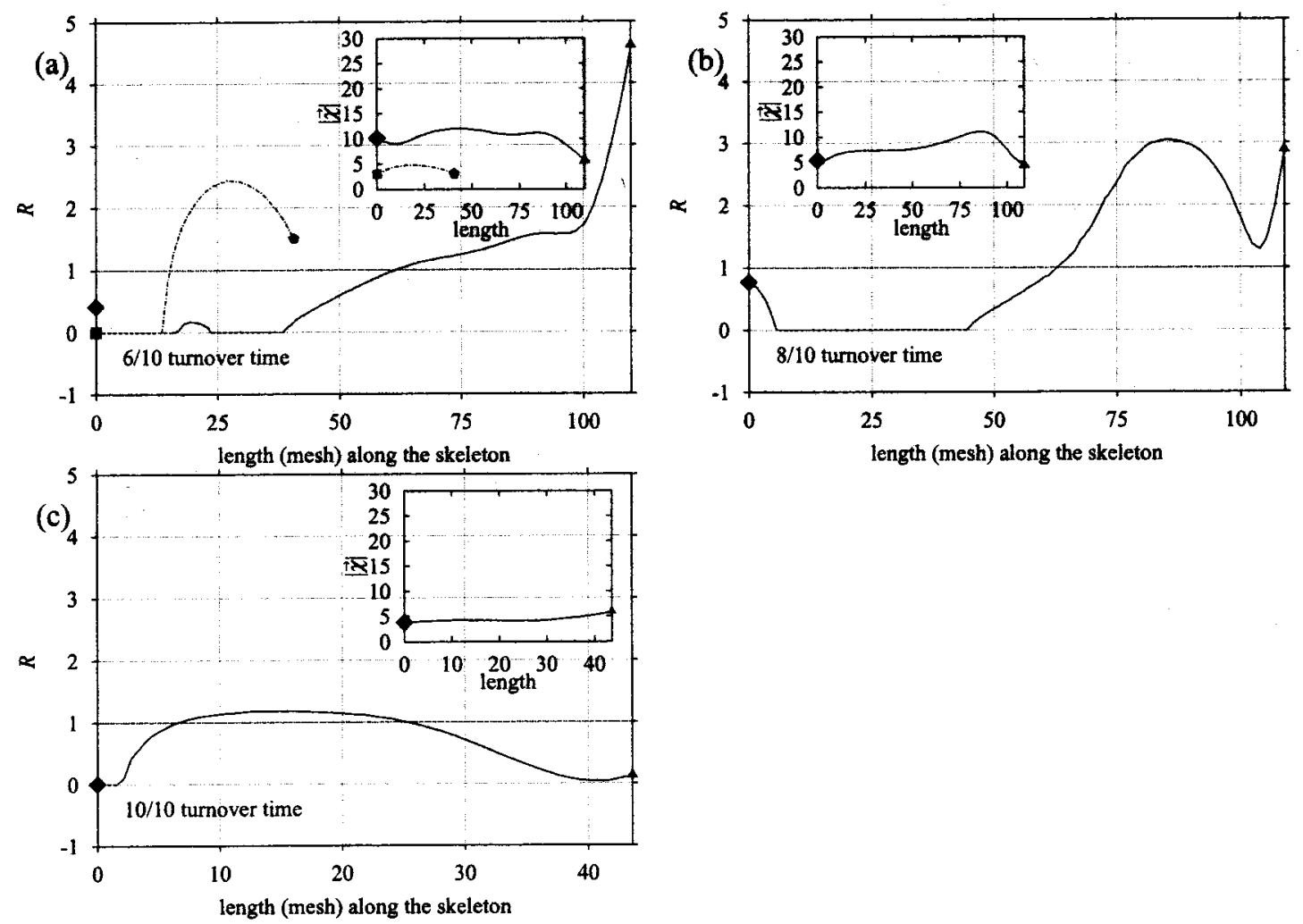

FIG. 12. The later stage evolution of the ratio $R$ of the structure shown in Fig. 6. Insets: corresponding modulus of $T$-vorticity, $|\boldsymbol{\chi}|$. 

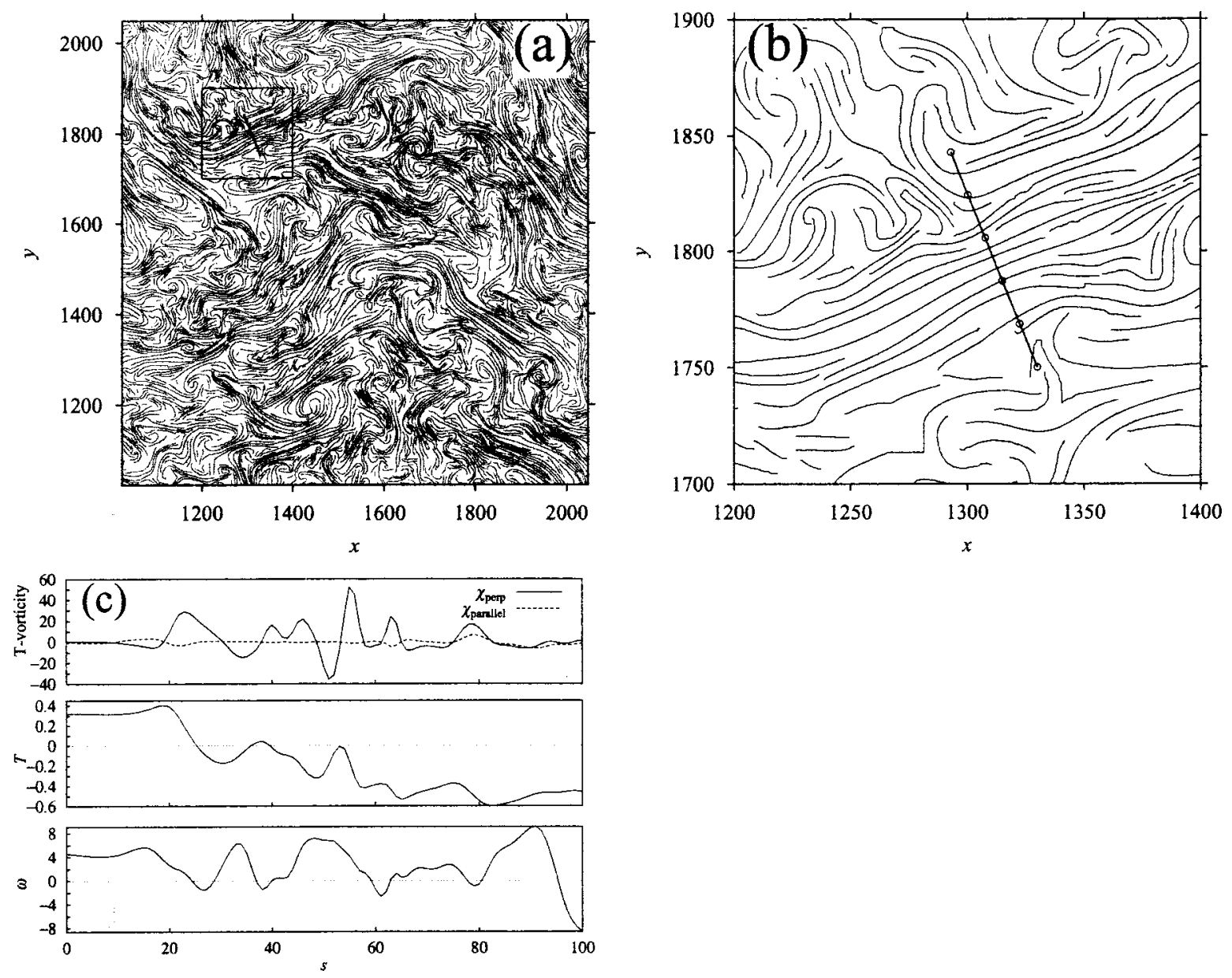

FIG. 13. (a) Skeletons obtained in the DNS. This figure contains $1024^{2}$ points out of $2048^{2}$ grid points. Long structures tend to form "bundles." (b) Enlargement of the square region $\left(200^{2}\right.$ grid points) in the upper left corner of (a). (c) $T$-vorticity, temperature and vorticity along the straight line shown in (b). The abscissa is the length in mesh units along the line: open circles in (b) from the right bottom end to the left top end point correspond to the points $s=0,20, \ldots, 100$. In the figure of $T$-vorticity, $\chi_{\text {perp }}$ and $\chi_{\text {parallel }}$ denote the components of the vector $\boldsymbol{\chi}$ perpendicular and parallel to the line, respectively.

The last term of Eq. (43) represents the vorticity induced by the buoyancy.

Now we discuss the realizability of the external flow, Eq. (32). If we claim that Eq. (33) is a steady solution of the barotropic two-dimensional vorticity equation,

$$
(\mathbf{U} \cdot \nabla) \Omega=\nu \Delta \Omega,
$$

where $\mathbf{U}=\left(\partial_{y} \Psi,-\partial_{x} \Psi\right)$. Then the equation for $f(x)$ is

$$
\frac{\nu}{A} f^{(4)}(x)+f(x) f^{(3)}(x)-f^{\prime}(x) f^{\prime \prime}(x)=0,
$$

where $f^{(j)}(x)$ denotes the $j$ th order derivative. Equation (45) has been studied by many authors as a model of a flow near a rigid wall. ${ }^{15}$ In Ref. 17, Chen and Okamoto, who were interested in the finite time blow-up of the solution, dealt with the time-dependent stream function $\Psi=-A y f(x, t)$ in a bounded domain and discussed the asymptotic behavior $(t \rightarrow \infty)$ of the solution to the equation for $f(x, t)$, the Proudman-Johnson equation. ${ }^{16}$ They showed that every solution decays to zero as $t \rightarrow \infty$ with homogeneous boundary conditions.

Here instead of solving Eq. (45), we assume that the flow, Eq. (32), is formed by a larger scale flow in the turbu- lence field. That is, from a practical point of view, a steady solution subjected to an external flow is no more than a local model of coherent structures in the turbulent field, so that the external flow need not satisfy Eq. (44). Thus it may be possible to add a forcing term describing an effect of the larger scale motions to Eq. (44). Denoting the forcing term by $G(x, y)$, the equation for $\Omega$ [or $f(x)]$ can be re-written as

$$
(\mathbf{U} \cdot \boldsymbol{\nabla}) \Omega=\nu \Delta \Omega+G .
$$

Then the steady-state equation for the total vorticity, Eq. (37), can be written as

$$
(\mathbf{u} \cdot \boldsymbol{\nabla})(\Omega+\widetilde{\omega})=\chi_{y}(x) \alpha g \sin \varphi+\nu \Delta(\Omega+\widetilde{\omega})+G,
$$

which yields the equation for the response vorticity $\widetilde{\omega}$,

$$
\frac{\nu}{A} \frac{d^{2} \widetilde{\omega}}{d x^{2}}+f(x) \frac{d \widetilde{\omega}}{d x}=\chi_{y}(x) \frac{\alpha g \sin \varphi}{A} .
$$

The solution, Eq. (43), is obtained from Eq. (48). By substituting $f(x)=x$, it is easily checked that the solutions Eqs. (42) and (43) contain Burgers vortex layer solutions.

In the following, we present two examples of $f(x)$ showing more than one structure. As the first example, we deal with an external flow, 


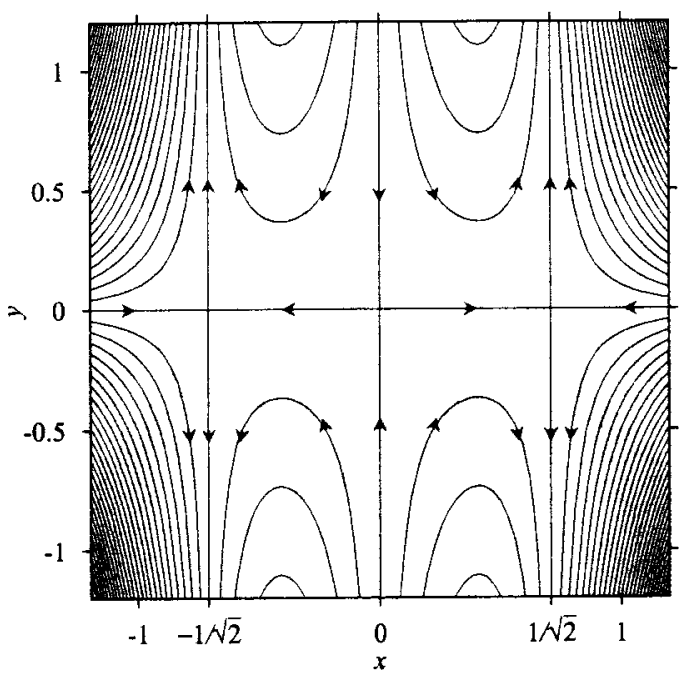

FIG. 14. Stream lines of an external flow $\Psi(x)=-y\left(4 x^{3}-2 x\right)$. The arrows denote the directions of the flow. Stagnation points are $(-1 / \sqrt{2}, 0),(0,0)$ and $(1 / \sqrt{2}, 0)$.

$$
f(x)=4 x^{3}-2 x
$$

which itself has vorticity $\Omega(x, y)=24 A x y$. The stream lines of the external flow is shown in Fig. 14. Here we assume an additional forcing $G(x, y)$ that keeps the external field, Eq. (49), steady. [Equation (46) prescribes the forcing as $G(x, y)=192 A^{2} x^{3} y$.] We impose the boundary conditions,

$$
\begin{aligned}
& \chi_{y}(x=0)=\chi_{0} \neq 0, \\
& \chi_{y}^{\prime}(x=0)=0, \\
& \widetilde{\omega}(x=0)=\widetilde{\omega}_{0}, \\
& \widetilde{\omega}(x=0)^{\prime}=\widetilde{\omega}_{0}^{\prime}=0 .
\end{aligned}
$$

If the field $f(x)$ is an odd function, taking the condition for vorticity, Eq. (53), is equivalent to assuming that the solutions are even. This results in the localization of vorticity around the origin. The solutions, Eqs. (42) and (43), are then reduced to
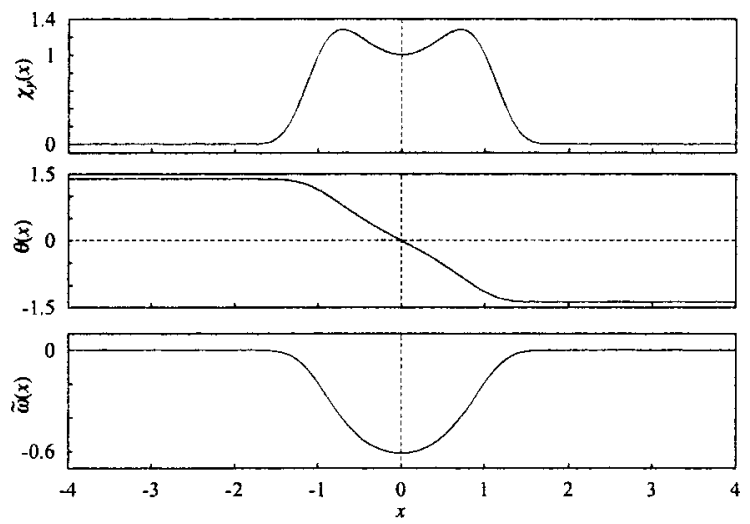

FIG. 15. The solutions for $\Psi(x)=-A y\left(4 x^{3}-2 x\right)$ : the $T$-vorticity Eq. (54), the corresponding temperature $\theta(x)$ and the response vorticity, Eq. (55). Here we set the parameters as $A=1, \kappa=1, \alpha g=1, \varphi=\pi / 4, \chi_{0}=1$, $\theta(x=0)=0$ and $\widetilde{\omega}_{0}=-e^{1 / 4} \sqrt{\pi}\left[1+(2 / \sqrt{\pi}) \int_{0}^{1 / 2} e^{-\zeta^{2}} d \zeta\right] /(4 \sqrt{2})=-0.61$.

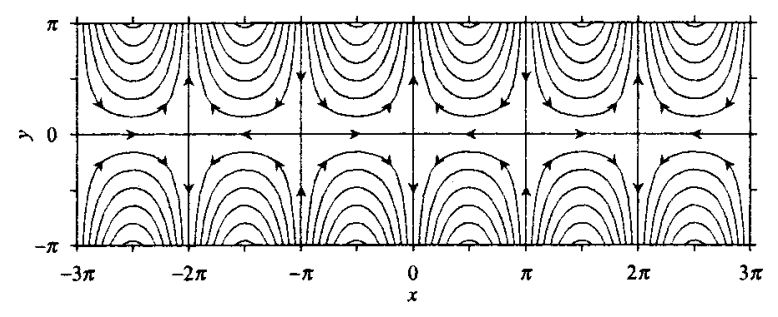

FIG. 16. Stream lines of an external flow $\Psi(x)=-y \sin x$. The arrows denote the directions of the flow. Stagnation points are $(n \pi, 0), n=0, \pm 1$, $\pm 2, \ldots$.

$$
\begin{aligned}
\chi_{y}(x) & =\chi_{0} e^{-(A / \kappa)\left(x^{4}-x^{2}\right)} \\
\widetilde{\omega}(x) & =\widetilde{\omega}_{0}+\chi_{0} \frac{\alpha g \sin \varphi}{\kappa} \int_{0}^{x} \xi e^{-(A / \kappa)\left(\xi^{4}-\xi^{2}\right)} d \xi \\
& =\widetilde{\omega}_{0}+\chi_{0} \frac{e^{(A / 4 \kappa)} \alpha g \sin \varphi}{2 \kappa} \int_{0}^{x^{2}} e^{-(A / \kappa)(\zeta-1 / 2)^{2}} d \zeta
\end{aligned}
$$

The constant $\widetilde{\omega}_{0}$ is determined so that the response vorticity vanishes at infinity, i.e.,

$$
\widetilde{\omega}_{0}=-\chi_{0} \frac{e^{(A / 4 \kappa)} \alpha g \sin \varphi}{2 \kappa}\left[\frac{1}{2} \sqrt{\frac{\kappa \pi}{A}}+\int_{0}^{1 / 2} e^{-(A / \kappa) \zeta^{2}} d \zeta\right] .
$$

These solutions are plotted in Fig. 15. There are two bumps in $\chi_{y}$. This solution catches features seen in Fig. 13(c) around $s=40$.

As another example, let us consider the periodic flow,

$$
f(x)=\sin x .
$$

This flow has vorticity $\Omega=-A y \sin x$. The corresponding forcing $G(x, y)$ is then given by $\nu A y \sin x$. The streamlines of this external flow Eq. (57) are shown in Fig. 16, where stagnation points are located periodically. We impose the boundary conditions, Eqs. (50)-(53). In particular we take $\widetilde{\omega}_{0}=0$ in this case. Then the general solutions, Eqs. (42) and (43), are expressed as

$$
\begin{aligned}
& \chi_{y}(x)=\chi_{0} e^{-(A / \kappa)(1-\cos x)}, \\
& \widetilde{\omega}(x)=\chi_{0} \frac{\alpha g \sin \varphi}{\kappa} \int_{0}^{x} \xi e^{-(A / \kappa)(1-\cos \xi)} d \xi,
\end{aligned}
$$

which are plotted in Fig. 17. The temperature shows the staircase-like behavior of $\theta(x)$. As a result of that a row of structures is seen. The solution, Eqs. (58) and (59), can be useful to model the small wavy behavior embedded on a large temperature front, which is similar to the one shown in Fig. 13(c). However, in Fig. 13(c) the strong peak of temperature around $s=55$ makes it rather hard to see this overall trend clearly.

\section{CONCLUDING REMARKS}

We have examined a class of steady solutions to the two-dimensional Boussinesq approximation equations. We believe that these solutions well describe coherent structures observed in the DNS of 2-DFC. Under an extended stagna- 

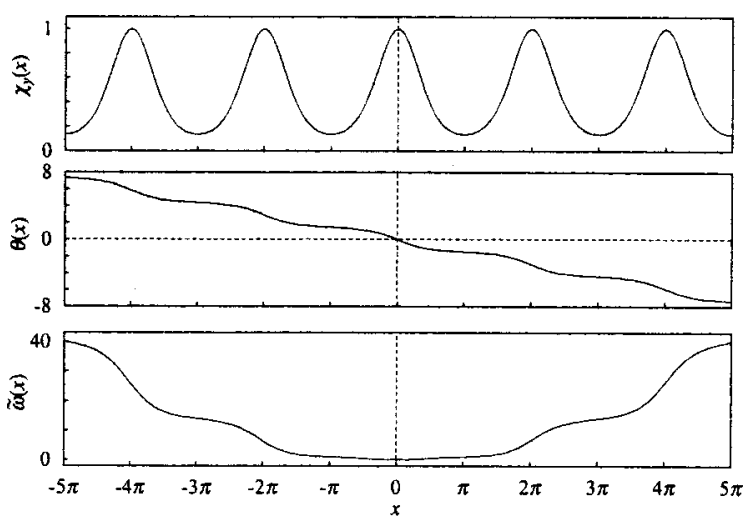

FIG. 17. A solution for $\Psi(x)=-A y \sin x$ : the $T$-vorticity, Eq. (58), the corresponding temperature $\theta(x)$ and the response vorticity, Eq. (59). Here we set the parameters as $A=1, \kappa=1, \alpha g=1, \varphi=\pi / 4, \chi_{0}=1, \theta(x=0)=0$ and $\widetilde{\omega}_{0}=0$.

tion flow $\Psi=-A y f(x)$, we obtained steady solutions of $T$-vorticity and vorticity. The steady $T$-vortex layers are maintained by the balance between the strain of an external stagnation flow and the diffusion. When $f(x)=x$, the Burgers vortex layer solutions are obtained, which model well individual coherent structure obtained in DNS. When $f(x)$ $\neq x$, the $T$-vortex solution can have multiple bumps, which can be a model of the collective behavior of the coherent structures seen in the DNS. So far we have taken for granted the independence of an external flow and a response. This view is reasonable if we focus on local behavior of the coherent structures. Indeed the local shape of the structures are quite similar to the steady solutions. Thus it is suggested that the characteristic time scale of the external strain field are well separated from that of structure's motions.

The role of the coherent structures in statistical properties of turbulence, which is expected to be universal, is still an open question. Since the coherent structures have quite long correlation length comparable to the scale of the entropy (temperature variance) containing range, their existence may significantly break the locality hypothesis. However, the authors have examined the relative diffusion in 2-DFC turbulence recently and found that the coherent structures rather play an essential role in keeping the locality. These contradicting characteristics of the coherent structures make research on turbulence challenging.
We believe that coherent structures are universal and essential ingredients of turbulence. Extensive researches on coherent structures are therefore required.

\section{ACKNOWLEDGMENTS}

We are grateful to the anonymous referees for the constructive criticism. This work has been partially supported by a Grant-in-Aid for Science Research on Priority Areas (B) from the Ministry of Education, Culture, Sports, Science and Technology of Japan. Numerical computation in this work was done at the Yukawa Institute Computer Facility.

${ }^{1}$ U. Frisch, Turbulence: The Legacy of A. N. Kolmogorov, 2nd printing (Cambridge University Press, Cambridge, 1996), Sec. 8.9.

${ }^{2}$ Z. Warhaft, "Passive scalars in turbulent flows," Annu. Rev. Fluid Mech. 32, 203 (2000).

${ }^{3}$ A. Celani, A. Lanotte, A. Mazzino, and M. Vergassola, "Fronts in passive scalar turbulence," Phys. Fluids 13, 1768 (2001).

${ }^{4}$ A. Celani, A. Lanotte, A. Mazzino, and M. Vergassola, "Universality and saturation of intermittency in passive scalar turbulence," Phys. Rev. Lett. 84, 2385 (2000).

${ }^{5}$ V. S. L'vov, "Spectra of velocity and temperature fluctuations with constant entropy flux of fully developed free-convective turbulence," Phys. Rev. Lett. 67, 687 (1991).

${ }^{6} \mathrm{~S}$. Toh and E. Suzuki, "Entropy cascade and energy inverse transfer in two dimensional convective turbulence," Phys. Rev. Lett. 73, 1501 (1994).

${ }^{7}$ A. Celani, A. Mazzino, and M. Vergassola, "Thermal plume turbulence," Phys. Fluids 13, 2133 (2001).

${ }^{8}$ D. Biskamp, K. Hallatschek, and E. Schwarz, "Scaling laws in twodimensional turbulent convection,” Phys. Rev. E 63, 045302 (2001).

${ }^{9}$ For two-dimensional barotoropic vorticity, see S. Kida and M. Yamada, "Singularity and energy spectrum in a two-dimensional incompressible inviscid flow," in Turbulence and Chaotic Phenomena in Fluids, edited by T. Tatsumi (Elsevier Science, New York, 1984), p. 275.

${ }^{10}$ P. Constantin, "Geometric statistics in turbulence," SIAM Rev. 36, 73 (1994).

${ }^{11}$ T. S. Lundgren, "Strained spiral vortex model for turbulent fine structure," Phys. Fluids 25, 2193 (1982).

${ }^{12}$ M. Holzer and E. D. Siggia, "Turbulent mixing of a passive scalar," Phys. Fluids 6, 1820 (1994); erratum, ibid. 7, 1519 (1995).

${ }^{13} \mathrm{H}$. Miura and S. Kida, "Identification of tubular vortices in turbulence," J. Phys. Soc. Jpn. 66, 1331 (1997).

${ }^{14}$ A. Mazzino (private communication, 2000).

${ }^{15} \mathrm{G}$. K. Bachelor, An Introduction to Fluid Dynamics (Cambridge University Press, Cambridge, 1967), Sec. 5.5, (b).

${ }^{16}$ I. Proudman and K. Johnson, "Boundary-layer growth near a rear stagnation point," J. Fluid Mech. 12, 161 (1962).

${ }^{17} \mathrm{X}$. Chen and H. Okamoto, "Global existence of solutions to the Proudman-Johnson equation,” Proc. Jpn. Acad. 76, 149 (2000). 